\title{
Perfuração Retal Incompleta após Enema Opaco: Relato de Caso
}

\author{
Incomplete Rectal Perforation After Barium Enema: A Case Report
}

\author{
RODRIGO ROCHABATISTA'; CARLOS ALBERTOTORRES DE CASTRO ${ }^{1}$; ANDRÉ LUIGI PINCINATO²; IDBLAN \\ CARVALHODEALBUQUERQUE ${ }^{3}$; GALDINO JOSÉ SITONIOFORMIGA ${ }^{4}$
}

\begin{abstract}
${ }^{1}$ Residente do Serviço de Coloproctologia do Hospital Heliópolis, FSBCP; ${ }^{2}$ Médico Assistente do Serviço de Coloproctologia do Hospital Heliópolis, FSBCP; ${ }^{3}$ Médico Assistente do Serviço de Coloproctologia do Hospital Heliópolis, TSBCP; ${ }^{4}$ Chefe do Serviço de Coloproctologia do Hospital Heliópolis, TSBCP.
\end{abstract}

\begin{abstract}
BATISTA RR; CASTRO CAT; PINCINATO AL; ALBUQUERQUE IC; FORMIGA GJS. Perfuração Retal Incompleta após Enema Opaco: Relato de Caso. Rev bras Coloproct, 2010;30(3): 347-351.

RESUMO: O enema opaco, apesar de atualmente apresentar indicações restritas, continua útil na propedêutica radiológica do cólon. A perfuração colorretal é a mais grave complicação do enema opaco, ocorrendo em 0,02 a 0,23\% dos exames realizados, com taxa de mortalidade de até $50 \%$. É relatado caso de paciente masculino, 40 anos, há dois meses apresentou dor anal intensa e sangramento durante a realização de enema opaco para investigação etiológica de constipação, com melhora espontânea após um mês. Ao exame proctológico constatou-se infiltração da margem anal, submucosa do canal anal e reto extraperitoneal por sulfato de bário, sem locais de perfuração. $O$ enema opaco trazido pelo paciente e a radiografia simples de pelve após dois meses do exame mostravam perfuração retal incompleta por sulfato de bário. Optado por observação clínica e intervenção cirúrgica se houver complicação. Atualmente o paciente segue em acompanhamento ambulatorial, permanecendo assintomático há 15 meses.
\end{abstract}

Descritores: Perfuração, enema, complicações, tratamento, observação.

\section{INTRODUÇÃO}

O enema opaco, apesar de atualmente apresentar indicações restritas, continua sendo muito útil na propedêutica radiológica do cólon. ${ }^{(1,2)}$

O estudo radiológico contrastado do cólon é um exame invasivo de fácil realização, podendo raramente levar a complicações catastróficas tais como a perfuração retal ou colônica, megacólon tóxico, septicemia, embolia venosa por bário e enchimento gastrintestinal retrógrado associado a vômitos e aspiração em crianças. ${ }^{(3-6)}$ A perfuração colorretal é a mais grave complicação do enema opaco, ocorrendo em 0,02 a $0,23 \%$ dos exames realizados, com taxa de mortalidade de até $50 \% .^{(2,4-8)}$

O objetivo deste trabalho é relatar um caso de perfuração incompleta do reto após enema opa- co, ocasionando a infiltração do bário na região anorretal.

\section{RELATO DO CASO}

C.J.P.A., masculino, 40 anos, há dois meses apresentou dor anal intensa e sangramento durante a realização de enema opaco para investigação diagnóstica de constipação intestinal. Referiu também grande dificuldade para realização do exame. Evoluiu com prurido e manutenção da dor em região perianal, com melhora espontânea dos sintomas há um mês.

No exame proctológico, à inspeção, apresentava na margem anal nodulação na posição lateral direita e placa mediana anterior, ambas de cor esbranquiçada, consistência fibroelástica, medindo aproximadamente $1,5 \mathrm{~cm}$ de diâmetro cada (figura 1);

Trabalho realizado no Serviço de Coloproctologia do Hospital Heliópolis, São Paulo/SP.

Recebido em 22/12/2009

Aceito para publicação em 25/01/2010 
ao toque retal, presença de infiltração submucosa circunferencial do canal anal, contíguo às lesões de margem anal, estendendo-se até $7 \mathrm{~cm}$ em posição mediana anterior e lateral direita e até $5 \mathrm{~cm}$ nos demais quadrantes; à anuscopia e retossigmoidoscopia, notava-se infiltração da submucosa do canal anal e reto extraperitoneal por sulfato de bário, sem locais de perfuração (figura 2).

O enema opaco trazido pelo paciente (figura 3) e a radiografia simples de pelve em posição ânteroposterior após dois meses do exame (figura 4) confirmaram a suspeita diagnóstica de perfuração retal incompleta por sulfato de bário, sendo visibilizado contraste em finas camadas longitudinais na parede retal, o qual representa dissecção entre a mucosa e a camada muscular do reto.

Optado por observação clínica e intervenção cirúrgica se houver complicação. Atualmente o paciente segue em acompanhamento ambulatorial, permanecendo assintomático há 15 meses.

\section{DISCUSSÃO}

A perfuração iatrogênica do reto durante o enema opaco é uma complicação rara de um procedimento comum e associada à alta taxa de mortalidade. ${ }^{(7,9-14)} \mathrm{O}$ primeiro relato de vazamento intraperitoneal de contraste foi feito por Rosenthal em 1916, e foi atribuído a uma perfuração do estômago. ${ }^{(15)}$ Em 1932, Himmelmann fez o primeiro relato de extravasamento de contraste durante o enema opaco. ${ }^{(16)}$

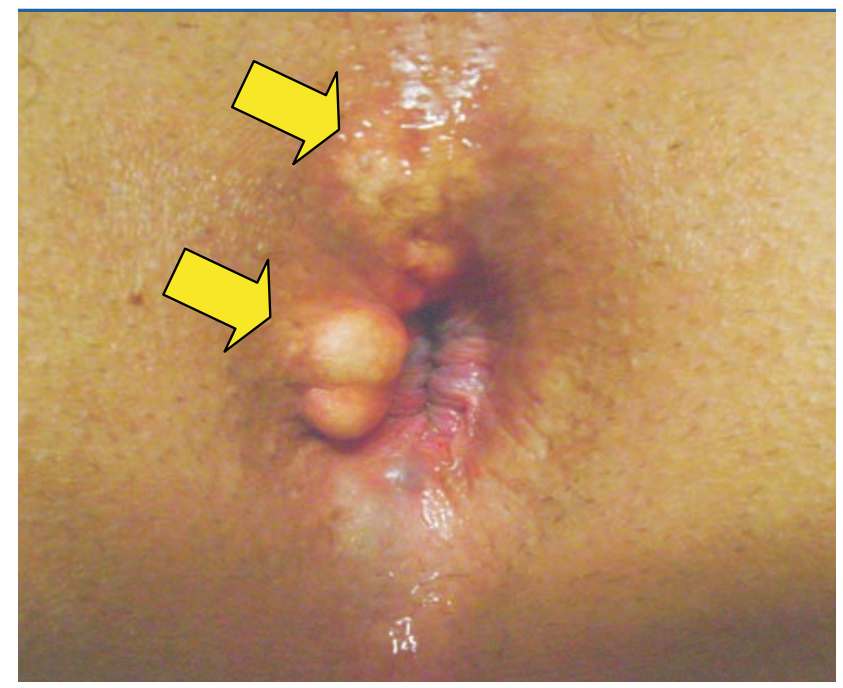

Figura 1 - Infiltração do anoderma por sulfato de bário.

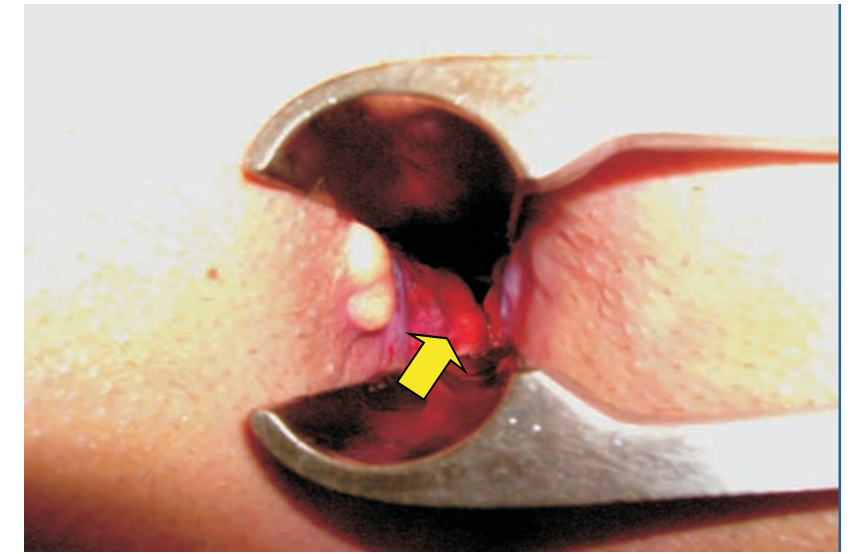

Figura 2 - Infiltração da submucosa por sulfato de bário.

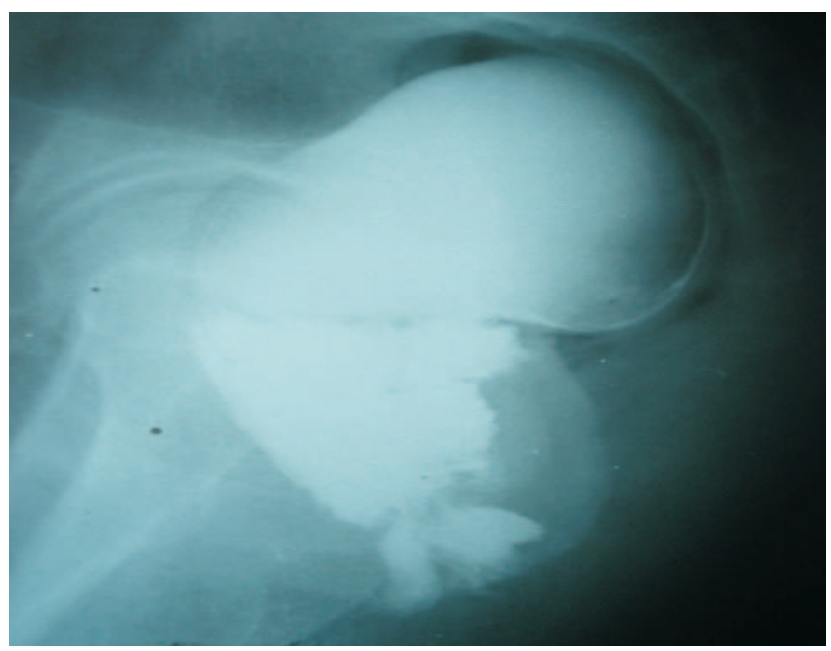

Figura 3 - Enema opaco mostrando a infiltração.

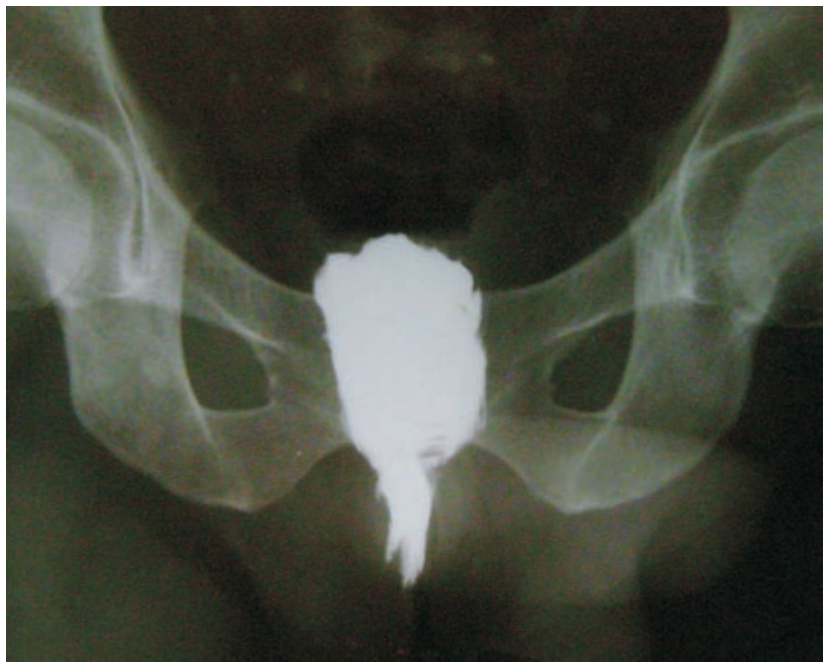

Figura 4-Radiografia simples de pelve três meses após a infusão do contraste. 
A etiologia das perfurações anorretais pode ser dividida em dois grupos, a iatrogênica e a secundária à fraqueza da parede colorretal. As perfurações iatrogênicas podem ocorrer por introdução forçada do cateter em direção à parede anterior do reto, pressão hidrostática intracolônica excessiva durante a injeção do contraste, hiperinsuflação do balão, insuflação do balão no canal anal, insuflação do balão ao nível da anastomose colorretal ou de lesões. O segundo grupo corresponde aos pacientes portadores de retocolite ulcerativa, doença de Crohn, diverticulite aguda, colite isquêmica ou infecciosa, neoplasia colorretal obstrutiva, estenose colorretal, fecaloma, antecedente de radioterapia pélvica, parasitose intestinal, biópsia ou polipectomia recente, idade avançada, corticoterapia, colostomia, fístula anorretal e fissura anal, os quais são fatores de risco que tornam estes pacientes mais susceptíveis à perfuração retal durante o enema opaco. ${ }^{(2,4,5,7,8,10-12,17-21)}$

Em 1939, Ault GW classificou as perfurações retais por enema opaco em cinco tipos: 1) perfuração do canal anal abaixo dos músculos elevadores do ânus; 2) perfuração incompleta; 3) perfuração para o retroperitôneo; 4) perfuração transmural para víscera adjacente; 5) perfuração livre para a cavidade peritoneal. ${ }^{(22)}$ Nosso paciente apresentou perfuração retal incompleta (segundo tipo) com infiltração anorretal. Ainda podemos considerar um sexto tipo, o intravasamento de sulfato de bário para as veias de drenagem do segmento colorretal acometido. ${ }^{(9,20)} \mathrm{A}$ infiltração anorretal é uma forma de perfuração incompleta que ocorre quando o sulfato de bário é forçado através da descontinuidade da mucosa retal, formando granulomas de bário na submucosa do reto e anoderma. ${ }^{(7)}$

As manifestações clínicas da perfuração podem ser diferentes de acordo com o tipo de lesão. Dor retal durante a instrumentação (introdução do cateter, durante ou após injeção do sulfato de bário ou insuflação de ar) deve sempre ser interpretado como risco de perfuração e por isso merece investigação e tratamento em caso de lesão. Entretanto, inicialmente a dor pode ser mínima ou ausente devido à pobre inervação sensitiva do reto, podendo ser tardia a manifestação clínica e geralmente associada à maior gravidade. ${ }^{(4,5,8,13,14,17,21)}$ Sangramento retal, enfisema subcutâneo, pneumoperitôneo ou pneumoretroperitôneo também são fortes indícios de perfuração retal. Poucas horas após o incidente, pode surgir febre, taquicardia, dor abdomi- nal, sinais de irritação peritoneal, seguidos por sepse grave de rápida evolução, principalmente nos casos de contaminação peritoneal grosseira por bário misturado às fezes. Nos casos de perfurações para o retroperitôneo, geralmente há vazamento apenas de ar, e por isso o curso clínico nestes casos costuma ser menos agressivo, sendo $50 \%$ dos casos assintomáticos. ${ }^{(2,4-6,8,11,13,14,20,21)}$

A colonoscopia ou retossigmoidoscopia deverá ser realizada apenas na dúvida diagnóstica ou em casos de tratamento conservador quando se deseja localizar a perfuração. Nos pacientes com indicação de tratamento operatório, o exame endoscópico não tem valor e pode agravar as complicações sépticas da perfuração. ${ }^{(4)}$

Na suspeita de perfuração retal durante a realização do enema opaco, a sonda retal ou cateter-balão deverá ser mantido até que ocorra drenagem da maior quantidade possível do contraste. O tratamento inicial consiste em: jejum, hidratação venosa, antibioticoterapia de amplo espectro e nutrição parenteral. (2,-4-6,10-13,21) $^{-}$

Os pacientes com perfuração retal incompleta, perfuração colorretal intraperitoneal ou retroperitoneal pequena, sem evidência de escape de contraste ou com melhora clínica durante observação por pouco tempo, podem ser tratados conservadoramente. ${ }^{(2,4-6,10-13,21)} \mathrm{O}$ tratamento cirúrgico nestes casos está indicado naqueles que não respondem ao tratamento conservador e nos casos de abscesso intramural. $\mathrm{Na}$ vigência de perfuração retroperitoneal ou intraperitoneal com grande extravasamento de contraste, ou perfuração para o interior de víscera adjacente, está indicado o tratamento cirúrgico para retirar a maior quantidade possível de bário através de lavagem, localizar e reparar o local da perfuração, seja através de sutura primária com derivação fecal proximal, ressecção da lesão com anastomose primária associada à derivação fecal proximal ou cirurgia de Hartmann, associado ou não à drenagem présacral. A escolha da técnica e tática operatória dependerá da condição clínica do paciente, das condições locais no intra-operatório e da experiência do cirurgião..$^{(2,4-6,10-13,19-21)}$ Nos pacientes com sepse, o tratamento agressivo do choque séptico com reposição de fluidos e drogas vasoativas será tão importante quanto a cirurgia precoce. As perfurações abaixo dos músculos elevadores do ânus com grandes lacerações de mucosa ou lesões esfincterianas, quando diagnosticadas precocemente, podem ser submetidas 
a reparo primário. Os abscessos anorretais devem ser drenados. ${ }^{(4,11,13,20,21)}$

A morbidade e mortalidade dos pacientes com perfuração colorretal por enema opaco se correlacionam com o tempo para o reparo da lesão. ${ }^{(5)}$ Os fatores de mau prognóstico são extravasamento excessivo do sulfato de bário, demora no diagnóstico, presença de fezes na cavidade peritoneal, mau preparo do cólon e idade avançada. ${ }^{(8)}$ No caso relatado não foi realizado tratamento devido a demora na procura médica pelo paciente e ausência de sintomas e complicações quando do diagnóstico.

Os pacientes que sobrevivem à perfuração colorretal por enema opaco podem evoluir com complicações tardias, tais como granuloma (baritoma, o qual simula um tumor), estenose retal, fístula, aderências intestinais e fibrose retroperitoneal com ou sem obstrução ureteral. ${ }^{(4,5,7,8,12,13,19-21)}$

O enema opaco é um exame útil, porém invasivo, que necessita de cuidado durante a sua realização, tais como reconhecer a anatomia da região anorretal com a ajuda do toque retal, introduzir a sonda retal até cinco centímetros acima da borda anal, insuflar o balão com até 400 mililitros e logo acima do canal anal, manter a coluna de contraste até um metro acima do paciente e evitar a realização do enema opaco por pelo menos duas semanas após biópsia ou instrumentação colorretal. $^{(4,20,21)} \mathrm{O}$ tratamento da perfuração retal deverá ser precoce, individualizado para cada tipo de lesão e na dependência das condições clínicas do paciente. Assim, essas medidas poderão reduzir a morbidade e mortalidade desta afecção.

\begin{abstract}
The barium enema, although present indications are currently restricted, is still useful on radiological workup in the colon. Colorectal perforation is the most serious complication of barium enema, occurring at 0.02 to $0.23 \%$ of examinations performed, with mortality rate of up to $50 \%$. We report the case of male patient, 40 years, two months ago had intense anal pain and bleeding during performing of barium enema for etiologic investigation of constipation, with spontaneous improvement after a month. On examination, there was cutaneus and submucosal infiltration of the anus and extraperitoneal rectum by barium sulfate, without perforation. The enema brought by the patient and the radiography of the pelvis after two months of the examination showed incomplete rectal perforation by barium sulfate. Performed clinical observation and surgical intervention only if arises complication. Currently, patient remains asymptomatic for 15 months.
\end{abstract}

Key words: Perforation, enema, complications, therapeutics, observation.

\section{REFERÊNCIAS}

1. De Zwart IM, Griffioen G, Shaw MP, Lamers CB, de Roos A. Barium enema and endoscopy for the detection of colorectal neoplasia: sensitivity, specificity, complications and its determinants. Clin Radiol 2001;56:401-9.

2. Tengrup I, Fork FT, Leandoer L. Deposition of barium sulphate outside the colon after barium enema examination. Eur J Surg 1997; 163: p 713-716.

3. Corman ML, Allison SI, Kuehne JP. Handbook of Colon \& Rectal Surgery. Lippincott Williams \& Wilkins 2006.

4. De Feiter PW, Soeters PB, Dejong CHC. Rectal perforations after barium enema: a review. Dis Colon Rectum 2005; 49(02): p 261-271.

5. Gebebou TM, Wong RA, Rappaport WD, Jaffe P, Kahsai D, Hunter GC. Clinical presentation and management of iatrogenic colon perforations. Am J Surg 1996; 172: p 454457.

6. Wang TK, Tu HH. Colorectal perforation with barium enema in the elderly: case analysis with the POSSUM scoring system. J Gastroenterol 1998; 33: p 201-205.
7. Lewis JW, Kerstein MD, Koss N. Barium granuloma of the rectum: an uncommon complication of barium enema. Ann Surg 1975;181(4): p 418-423.

8. Terranova O, Meneghello A, Battocchio F, Martella B, Celi D, Nistri R. Perforations of the extraperitoneal rectum during barium enema. Int Surg 1989; 74: p 13-16.

9. White JS, Skelly RT, Gardner KR, Laird J, Regan MC. Intravasation of barium sulphate at barium enema examination. Br J Radiol 2006; 79: p e32-35.

10. Fry RD, Shemesh EI, Kodner IJ, Fleshman JW, Timmcke AE. Perforation of the rectum and sigmoid colon during bariumenema examination - management and prevention. Dis Colon Rectum 1989; 32: p 759-764.

11. Eu KW, Seow-Choen F, Goh HS. Unusual rectal perforationan individualized approach to management. Singapore Med J 1994; 35: p 79-81.

12. Tadros S, Watters JM. Retroperitoneal perforation of the rectum during barium enema examination. Can J Surg 1988; 31(1): p 49-50.

13. Peterson N, Rohrmann CA, Lennard ES. Diagnosis and treatment of retroperitoneal perforation complicating the 
double-contrast barium-enema examination. Radiology 1982; 144: p 249-252.

14. Wolfe WG, Silver D. Rectal perforation with profuse bleeding following an enema: case report and review of the literature. Arch Surg 1966; 92(5): p 715-717.

15. Rosenthal E. Rontgenologish beobachtete magenperforatie. Berlin Klinische Wochenschift 1916; 53: p 945-947.

16. Himmelmann W. Ueber die perforation im bereich des magendarmtraktus bei und nach der roentgenbreipassage. Munch Med Wochenschr 1932; 79: p 1567-1571.

17. Tanswell IJ, Irfan K, Kossakowski T, Townson G. Rectal perforation in ulcerative colitis: complication of an enema tip. Gastrointest Endosc 2009; 69(2): p 344.

18. Chu FSK, Peh WCG, Chan FL, Cheung ANY. Rectal perforation after barium enema in a patient with cytomegalovirus colitis - a case report. Ann Acad Med Singapore 1993; 22(5): p 794-797.

19. Cordone RP, Brandeis SZ, Richman H. Rectal perforation during barium enema - report of a case. Dis Colon Rectum 1988; 31: p 563-569.
20. Vandendris M, Giannakopoulos X. Retroperitoneal barytoma. Urology 1981;17(4): p 358-359.

21. Rosenklint A, Buemann B, Hansen P, Baden H. Extraperitoneal perforation of the rectum during barium enema. Scand $\mathrm{J}$ Gastroent 1975; 10: p 87-90.

22. Ault GW. Perforation of the rectum with enema tips. Transactions of the Annual Meeting of the American Proctologic Society 1939; 40: p 203-211.

\section{Endereço para correspondência:}

GALDINO JOSÉ SITONIO FORMIGA

Serviço de Coloproctologia do Hospital Heliópolis

R. Cônego Xavier, 276 - Vila Nova Heliópolis 04231-030- São Paulo, SP

Tel.: 11- 2274-7600 (ramal 244)

Fax: 11-2247-7646

E-mail: gformiga@ig.com.br 